\title{
The Superconducting Extraction Magnet system EMC2 for the AGOR cyclotron.
}

\author{
H.G. KNOOPERS, H.J.G. KROOSHOOP and H.H.J. TEN KATE, \\ University of Twente, Applied Superconductivity Centre, Enschede, the Netherlands.
}

K. PIETERMAN and J.A.M. DAM, Cryocon b.v., Amersfoort, The Netherlands.

\begin{abstract}
A new superconducting extraction magnet system EMC2, has recently been installed in the AGOR cyclotron. It consists of two almost identical and slightly bent modules to accommodate the curved extraction beam. The coil system of each module consists of three coils located symmetrically around the median- plane, providing a main dipole field, a gradient field and a dipole correction field. The coil system is indirectly cooled by means of liquid helium flowing through channels located in the upper and lower part of both modules. In order to reduce the dissipation in the $4 \mathrm{~K}$ region due to beam losses, a $20 \mathrm{~K}$ radiation shield has been installed. Positioning of the extraction system to select a certain beam trajectory is provided by a guiding and feed-through system. This paper deals with the design and manufacturing of the superconducting and cryogenic parts of the EMC2 extraction system and the results of a successful test are presented.
\end{abstract}

\section{INTRODUCTION.}

The purpose of the AGOR project is to construct a compact cyclotron with superconducting coils which will be able to accelerate light as well as heavy ions [1]. The assembly and tests of the whole system is executed at INP in Orsay and is now in its final phase. After these tests the cyclotron will finally be installed at the KVI in Groningen for application in the field of nuclear physics research. The extraction system of the AGOR cyclotron consists of three separate elements. The first element has to be an electrostatic deflector due to the limited separation between successive turns at the entrance of the extraction system. The second element is an electromagnetic channel (EMC1) which will give a beam separation in the order of $65 \mathrm{~mm}$ at the entrance of the third element, a superconducting electromagnetic channel (EMC2). The EMC2 superconducting extraction system with adjustment mechanism and separate cryostat is constructed as an unit that can be easily mounted in and out in the mid-plane of the cyclotron. Due to the limited height of $80 \mathrm{~mm}$ and partly even $60 \mathrm{~mm}$ between the upper and

Manuscript received September 20, 1993. lower part of the main split cryostat, the extraction system is located in the vacuum of the acceleration chamber.

For the Chalk River cyclotron [2] a prototype of a nonadjustable and not fully superconducting extraction channel, based on straight racetrack coils, was built. The extraction channel developed here is the first fully superconducting and adaptable extraction system constructed and taken into operation.

The superconducting extraction system described in this paper is constructed and assembled at the Applied Superconductivity Centre, part of the Low Temperature Division at the University of Twente.

\section{COIL SYSTEM.}

The superconducting extraction channel consists of two almost identical modules indicated as EMC2.1 and EMC2.2. Both channel modules are bent with a different radius. The magnetic centre of the EMC2.1 has a radius of $1006.3 \mathrm{~mm}$ and the corresponding radius of the EMC2.2 is $1116.3 \mathrm{~mm}$. The angular extraction of both modules are respectively $17.158^{\circ}$ and $16.258^{\circ}$. Each module contains a

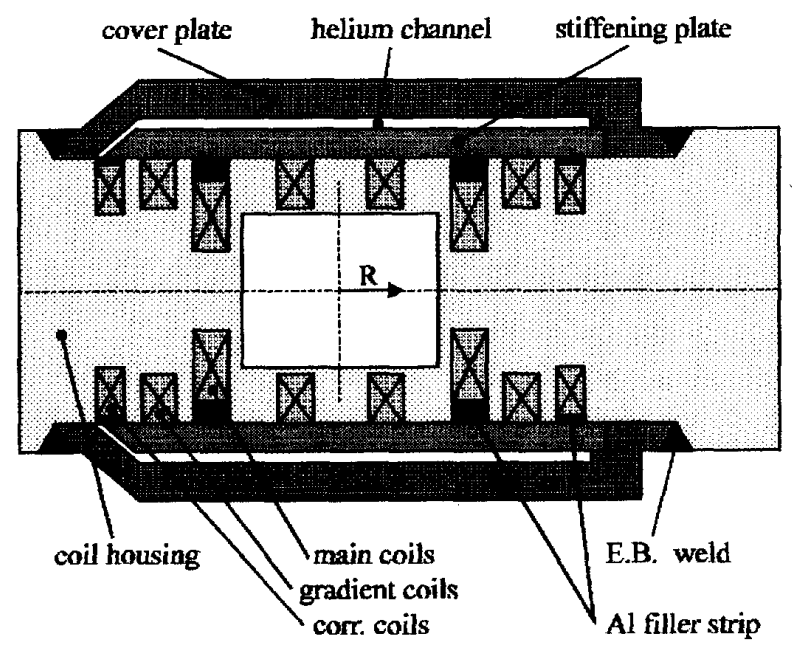

Fig. 1. A cross-section of the EMC2 superconducting extraction magnet. 
TABLE 1

Characteristic data of the EMC2 conductor.

\begin{tabular}{ll}
\hline Diameter & $0.500 \mathrm{~mm}$. \\
Diameter (bare) & $0.443 \mathrm{~mm}$. \\
Insulation & PEI \\
Number of filaments & 450. \\
Filament diameter & $11.4 \mathrm{~mm}$. \\
Twist pitch & $7 \mathrm{~mm}$. \\
RRR & 170. \\
Superconductor & $\mathrm{NbTi}$. \\
Cu/Sc & 2.4 \\
Ic and Jc a $2 \mathrm{~T}$ & $226 \mathrm{~A}$ and $5002 \mathrm{~A} / \mathrm{mm}^{2}$ \\
& $131 \mathrm{~A}$ and $2900 \mathrm{~A} / \mathrm{mm}^{2}$ \\
\hline
\end{tabular}

*Temperature $4.2 \mathrm{~K}$. Ic criterion $0.1 \mu \mathrm{V} / \mathrm{cm}$.

main coil, a gradient coil and a correction coil which are energised separately. The three coil systems provide the required field profile with an induction level up to $0.4 \mathrm{~T}$ in the bore of the extraction channel and with a very small magnetic field disturbance in the acceleration chamber $[3,4]$. The location of the coils with respect to the magnetic centre of the modules is shown in Fig. 1. The wire characterised in Table 1 is used for all coils in the extraction system EMC2. The extraction system operates in the main cyclotron field with an induction between $1.7 \mathrm{~T}$ and $4.1 \mathrm{~T}$. For each particular particle beam a specific current adjustment of each extraction coil is needed. The worst operational point is obtained at a main coil current of $80 \mathrm{~A}$ at a background field of $4 \mathrm{~T}$.

Coil winding technique: All superconducting coils of the system are wet wound on Al-moulds using the ARALDIT F epoxy system. Only in the outermost parts of the main coil ends $\mathrm{Al}_{2} \mathrm{O}_{3}$ is applied additionally as filler, to prevent crack formation under thermal shocks (about $50 \%$ filling factor). After the prescribed heat treatment the coils are removed from the mould.

The number of turns of the coils and the corresponding cross sections are listed in Table 2. Due to the limited space in the vertical direction, the cross sections of the main coil at the crossovers are $20 \times 3 \mathrm{~mm}^{2}$. In Fig. 2 the geometry of the coil ends is illustrated.

Insulation technique: Ground insulation of the coils is obtained by wetting the coils with STYCAST $2850 \mathrm{FT}$ after

TABLE 2

Coil turns per module and cross-sections.

\begin{tabular}{lcc}
\hline Coil & Number of turns & Cross-section $\left[\mathrm{mm}^{2}\right]$ \\
\hline Main & $2 \times 192$ & $10 \times 5$ \\
Gradient & $4 \times 125$ & $7 \times 5$ \\
Correction & $2 \times 100$ & $7 \times 5$ \\
\hline
\end{tabular}

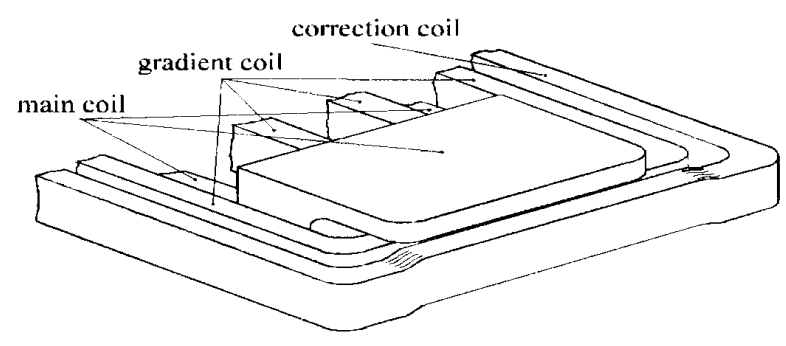

Fig. 2. Impression of the coil end geometry.

which they are wrapped in a KAPTON shcet. After completion, the insulated coil is wetted with STYCAST and inserted in the coil housing. The crossovers of the main coils are covered with an extra (Boron free) glass-cloth layer.

\section{CONSTRUCTION DETAILS.}

Coil housing: Due to the principal advantages of high thermal conductivity and low mass density, aluminium $1050 \mathrm{~A}$ is used for the EMC2 extraction system. The coil housing is manufactured out of one block of aluminium. The curved bore is obtained by spark erosion after which the slots for the coil systems are milled in the coil housing. After insertion of the correction and the main coils, aluminium $1050 \mathrm{~A}$ filler strips with a thickness of respectively $1 \mathrm{~mm}$ and $3 \mathrm{~mm}$ are glued with STYCAST 2850 FT on top of these coils.

A stiffening plate is glued and bolted to the coil housing to reduce the stress in the wall between the gradient coils and the bore. The cover plates are welded to the coil housing using electron beam (E.B.) welding. The penetration depth of the weld is about $5 \mathrm{~mm}$.

Cryogenic system: The superconducting coils are indirectly cooled by helium via the coil housing. Helium channels are located at the upper and lower part of the coil housing (see figure 1). The inlet and outlet connections of the coolant are placed at the end of the coil housing where both modules are joint together through a hinge connection. The inlet tube is located at the lower part of the coil housing. Helium will flow from the lower to the upper part through two holes, drilled at the opposite side to the inlet. The cooling channels of both modules are connected in series. The helium content of one coil module is about 0.1 litre.

Electrical connections: All corresponding coils in the EMC2.1 as well as in the EMC2.2 are connected in series. The electrical connections of the corresponding coils between the modules as well as those between the modules and the coaxial vapour cooled current leads in the helium reservoir, are located in the helium transfer lines. The diameter of the multifilament NbTi conductor used for these 
external connection leads is $0.90 \mathrm{~mm}$. The critical current at $3 \mathrm{~T}$ is $404 \mathrm{~A}$. These leads are covered with an extra Teflon (PTFE) insulation tube.

Inner thermal shield: To reduce the dissipation in the coil housing due to beam losses, an inner thermal shield is inserted in the bore. A photograph of such a shield is shown in Fig. 3. The inner shields are cooled in series to a temperature of $20 \mathrm{~K}$ by He-gas flowing trough six copper tubes of $\oslash 3.2 \mathrm{~mm}$ soldered onto a copper tube with a cross section of $25 \times 13 \mathrm{~mm}^{2}$. The side wall thickness of this rectangular tube is $4.5 \mathrm{~mm}$ leaving a beam aperture of $16 \mathrm{~mm}$ in radial direction and $12 \mathrm{~mm}$ in axial direction. Silver soldering is used for the connections of the six cooling tubes into the $\mathrm{He}$ - in and outlet gas connectors as well as for the Cu-AISI transition (to the bellows). All other connections for construction and/or to improve the heat conduction to the He gas are realised with $60 \mathrm{Sn} / 40 \mathrm{~Pb}$ solder. Positioning of the inner shields in the bore of the coil housing is obtained by AISI-strips connecting the inner shields to the coil housing at the beam entrance and exit side.

The beam loss is estimated to be $10 \mathrm{~W}$ per module. However, due to the application of the inner shields this dissipation is reduced to $1 \mathrm{~W}$ at the coil housing.

Connecting flanges: Each module of the superconducting extraction system is suspended by two thermal bridges to a connecting flange. Each thermal bridge consists of two AISI 304 pieces connected by a glass- epoxy tube with a wall thickness of $5 \mathrm{~mm}$. The connecting flanges are part of the background radiation (outer) shields. Copper $\mathrm{E}-\mathrm{Cu} 57$ is used for the construction of these flanges. Cooling will occur by means of He-gas, withdrawn from the 13 bar $80 \mathrm{~K}$ cooling circuit of the liquefier, flowing through copper cooling pipes which are soldered to these flanges. The connecting flanges are joined together through a hinge connection. Three swivel joints bolted to the opposite side of these flanges couple the modules to a guiding and feed-

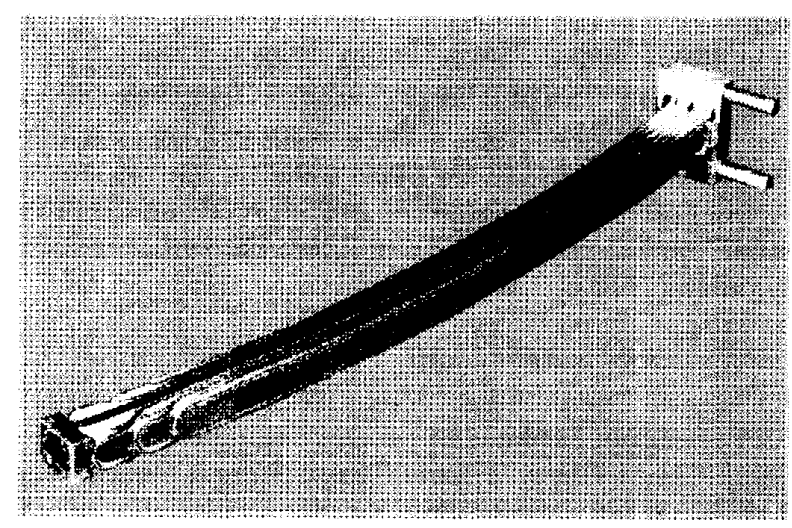

Fig. 3. Photograph of an inner shield. through system for positioning and adapting the shape of the channel to the trajectory of the extracted beam. The maximum displacement that can be adjusted is $\pm 6 \mathrm{~mm}$.

The outer shields that enclose the modules are constructed from Sf-Cu plate with a thickness of $2 \mathrm{~mm}$. These shields are assembled from parts that are joined together by silver soldering. Cooling is obtained through pressed metal contact around the bolts where the shields are connected to the connecting flanges. The surface of the shields are coated with a $2 \mu \mathrm{m}$ thick layer of gold. The heat conduction from the $80 \mathrm{~K}$ outer shields into the $4 \mathrm{~K}$ system through the supporting system is $0.8 \mathrm{~W}$ per module.

In Fig. 4 a photograph is presented of the described extraction system.

\section{TEST RESULTS}

Quench currents: No striking differences in the performance of both modules are observed. Therefore only the results of EMC2.2 are depicted. Fig. 5 shows the quench current of the coil system as function of the applied transverse field. A linear degradation with the field is observed. At $4 \mathrm{~T}$ a quench current in the main coils of $143 \mathrm{~A}$ is found. Referring to the worst operational current setting mentioned before, the operational current to quench current ratio is $56 \%$ maximum.

The quench currents are obtained after repeated quenching. The quench sequence is shown in Fig. 6. A small training effect is observed in all coil systems as could be expected with this type of coils. The plateau value, however. is reached only in a few steps. After reversing the current direction in the coils, fewer steps are necessary to attain the final value. After another reversal of the coil current. the maximum quench current is obtained in the first step.

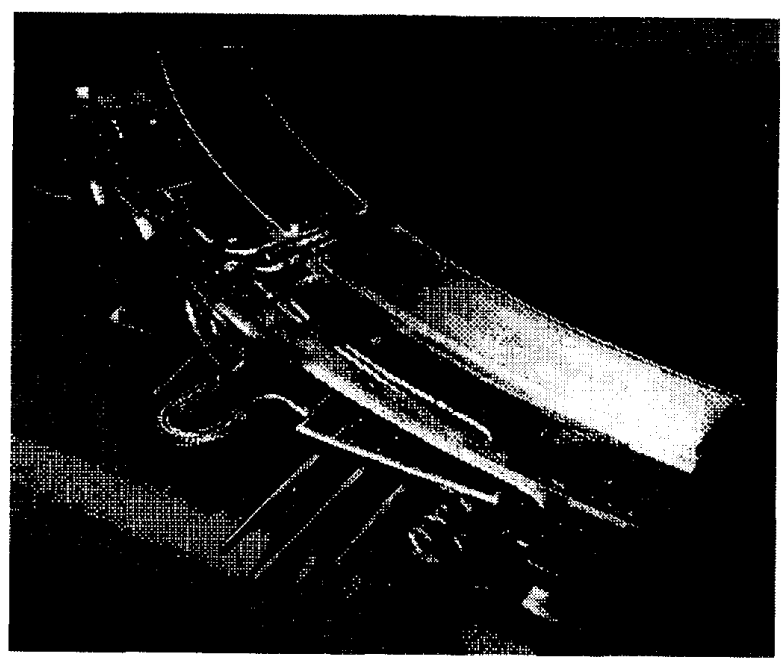

Fig. 4. Photograph of the extraction system EMC2. 


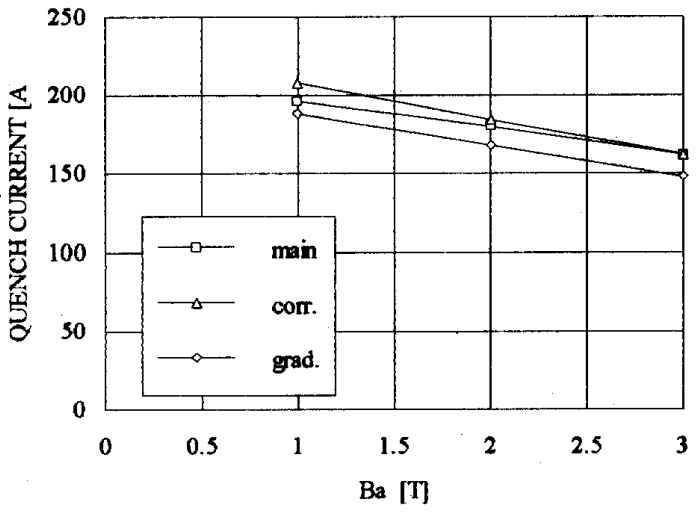

Fig. 5. Quench current vs. the applied field.

Stability: In order to determine the stability of the coil system against a local heat release, two heaters were placed on the walls inside the bore of the coil module. The modules were immersed in a helium bath. However, the coolant was exclude from the bore so cooling was obtained by conduction of the heat to the circumference of the module. All three coil systems were tested separately. Fig. 7 shows the quench current of the coil system as a function of the dissipation in the heater. The applied field is $3 \mathrm{~T}$. Up to a continuous dissipation of $10 \mathrm{~W}$, the quench current is greater than 140 A for all coil systems.

\section{CONCLUSION.}

As expected, a small training effect was observed in all three coil systems. However, the first quench current already

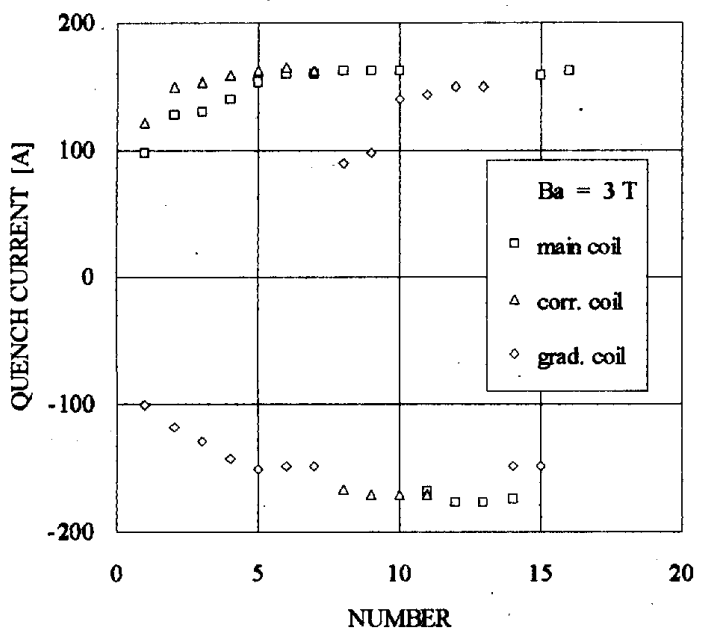

Fig. 6. Quench sequence of the EMC2.2 coils.

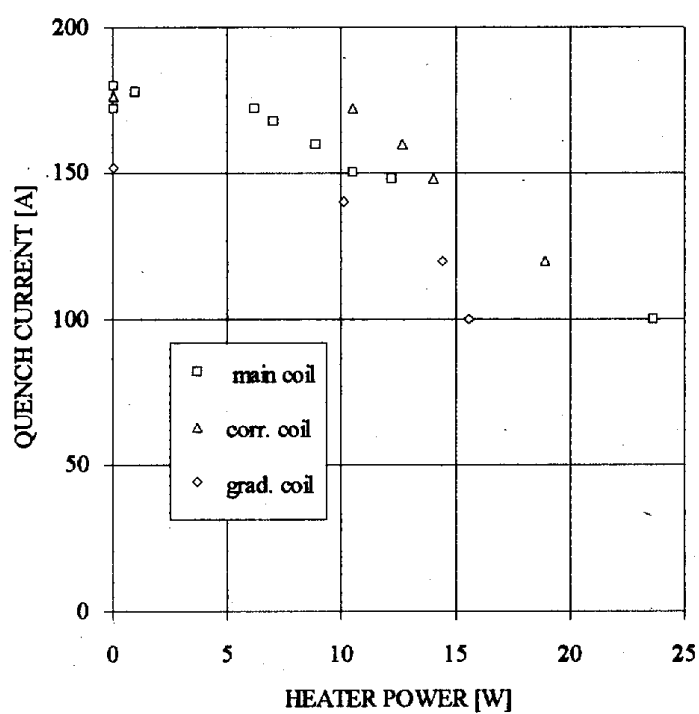

Fig. 7. Quench current as function of the dissipation in the heaters at a background field of $3 \mathrm{~T}$.

exceeds the maximum current during operation in the cyclotron. To reach the plateau current only a few quench steps were necessary. At a background field of $3 \mathrm{~T}$ the maximum current in the gradient coils is $150 \mathrm{~A}$ and in the main and correction coils $160 \mathrm{~A}$.

The most unfavourable operational current to quench current ratio that will occur is $56 \%$.

In a background field of $3 \mathrm{~T}$ the quench currents in the gradient coils, correction coils and main coils reduce to $100 \mathrm{~A}$ at a continuous heat release of respectively $15 \mathrm{~W}$, $21 \mathrm{~W}$ and $23 \mathrm{~W}$ at a spot in the bore of the coil housing.

To conclude, the test results are good and the system operates according to its specifications. The successful test of this unique, fully superconducting and fully adaptable extraction system completes the project and the system is now taken into operation.

\section{REFERENCES}

[1] S. Gales, "AGOR, a superconducting cyclotron for light and heavy ions", Proc. 11th Int. Conf. on Cyclotrons and their applications, Tokyo, 1986, p. 184 .

[2] C.R.Hoffman, J.F.Mouris and D.R.Proulx." Design and test of prototype superconducting extraction channel modules for the Chalk River superconducting cyclotron", Proc. 10th Int. Conf. on Cyclotrons and their applications, 1984, pp.222-225.

[3] S. Gustafsson, "A superconducting electromagnetic channel for AGOR", Proc. 12th Int. Conf. on Cyclotrons and their applications, Berlin 1989, pp.354-357.

[4] K. Pieterman, J.M.A. Dam, G. Levy, C. Commeaux, "A design study for a superconducting extraction element for AGOR", Proc. 12th Int. Conf. on Cyclotrons and their applications, Berlin 1989, pp.358-360. 